\title{
SER CHINO EN BUENOS AIRES: HISTORIA, MORALIDADES Y CAMBIOS EN LA DIÁSPORA CHINA EN ARGENTINA*
}

\author{
Luciana Denardi ${ }^{* *}$ \\ Universidad Nacional de San Martín - Argentina
}

Resumen: Este artículo se propone realizar una descripción de las particularidades de la comunidad china y taiwanesa en Buenos Aires. Recorre la historia de la migración china y taiwanesa hacia Argentina y los cambios que se están registrando en los últimos años, con la nueva posición de China en la economía mundial. Proponemos que la diáspora china y taiwanesa pueden ser pensadas como "puentes", ya que los taiwaneses se re identifican como "chinos" alegando una pertenencia ancestral al legado cultural chino, y los chinos continentales están ocupando el lugar asignado por el estado chino para sus diásporas: ser un eslabón en el proceso de expansión económica chino, intermediando entre la sociedad de recepción y la madre patria.

Palabras clave: diáspora china, diáspora taiwanesa, posiciones morales, sentidos de ser chino.

Abstract: This article proposes a description of the peculiarities of Chinese and Taiwanese community in Buenos Aires. It traces the history of Chinese and Taiwanese migration to Argentina and the changes that are occurring in recent years, with the new position of China in the world economy. We propose that the Chinese and Taiwanese diaspora can be understood of as "bridges", since Taiwanese re identify themselves as "Chinese" because off a cultural belonging to a Chinese legacy, and

\footnotetext{
* Los datos utilizados en este artículo surgen del trabajo de campo etnográfico en curso para la realización de la tesis de doctorado. Agradezco a Alejandro Grimson y Gustavo Ng, quienes me autorizaron a utilizar datos de la investigación que realizamos juntos perteneciente al proyecto "Migraciones asiáticas en América Latina y sus relaciones transnacionales” a cargo de Alejandro Portes, cuyos resultados se plasmaron en Grimson, Denardi y Ng (2014).

** Doctoranda en Antropología Social (becaria doctoral de la UNSAM). Contacto: lucianadenardi@gmail.com.
} 
mainland Chinese are taking the place assigned by the Chinese state to their diasporas: be a link in the process of Chinese economic expansion, mediating between the receiving society and the motherland.

Keywords: being Chinese, Chinese diaspora, moral positions, Taiwanese diaspora.

\section{Introducción}

Desde el momento en que la República Popular China ${ }^{1}$ comenzó su política de apertura a la economía mundial en 1979 (go out policy), ha logrado erigirse como una de las potencias mundiales en la primera década del siglo XXI, proceso en el que fortaleció o generó redes con países de América Latina y el Caribe principalmente. Las empresas transnacionales asiáticas y fundamentalmente las inversiones provenientes de China, no sólo vienen a América Latina en busca de materias primas e insumos sino que además tienen como objetivo ampliar los mercados en el exterior para hacer de sus firmas locales marcas globales (Moneta; Cesarín, 2012).

Consideramos que, este auge económico ha sido acompañado por una renovación del lazo entre el Estado Nación y sus diásporas, que pasaron del no reconocimiento a la integración en el entramado transnacional de su política económica. En este artículo expondremos, en primer lugar, las implicancias que reviste hablar de diáspora en el caso de los migrantes chinos. Luego, realizaremos un breve recorrido por las olas migratorias provenientes de China y Taiwán hacia la Argentina y la repercusión del conflicto entre ambos, en la vida cotidiana de sus migrantes. Así, nos aproximaremos a los diferentes sentidos de ser "chino" 2 en Buenos Aires, y a los cambios que se perciben en la diáspora ante el nuevo rol en la economía mundial de la República Popular China.

\footnotetext{
Nos referiremos a la República Popular China como RPC y como China. Mientras que para mencionar a la República de China también utilizaremos la denominación Taiwán.

2 Utilizaremos comillas para las palabras de nuestros informantes que surgen del trabajo de campo etnográfico. Las palabras en lengua inglesa o china se colocarán en cursivas y las citas bibliográficas se indicarán con cursivas y comillas.
} 


\section{Los overseas y la diáspora}

Hablar de diáspora china implica hablar de las "comunidades de ultramar” o su equivalente en inglés: overseas chineses. Según Barabantseva (2012, p. 81), en esta categoría se condensa la noción de continuidad y extensión de la nación china, más allá de sus fronteras geográficas. Esta extensión es referida por los migrantes, como el sentimiento de ser portadores de un legado ancestral en común y la nostalgia sobre China como el lugar donde se encontrará la sensación de completitud (Wu, 1991, p. 159) y de estar casa (Barabantseva, 2012, p. 96-97).

Según Barabantseva (2012), a lo largo de los años la diáspora overseas china ha ocupado diferentes roles. Desde que la Revolución de 1911 que dio por finalizada la era de las dinastías y comienzo a la República, fuera promovida por un grupo de estudiantes en Japón, la diáspora ocupó el rol de salvar a China. Luego de las reformas políticas y económicas de Deng Xiaoping, las comunidades de ultramar fueron invitadas a servir a la causa de la modernización nacional. Siguiendo las políticas de unificación con minorías étnicas al interior del territorio, el gobierno de la República Popular de China comenzó a promover políticas identitarias que intentan nacionalizar los migrantes chinos en una "unidad étnica transnacional” (Barabantseva, 2012, p. 80). Actualmente, se los convoca a construir una nueva y moderna nación posible gracias a las características que China le aporta al modelo socialista (Louie, 2000, p. 646).

A partir de estas características -la pertenencia a un legado cultural ancestral y la necesidad de servir a la nación-, entendemos que la diáspora china emerge como puente: "to be in diáspora is not only to traverse various cultural and national spaces, but also to erect bridges between here and there" (Zhang, B., 2006, p. 5). En este artículo analizaremos los sentidos de ser "chino" en Buenos Aires y las situaciones en las que la diáspora se convierte en un puente entre los chinos y/o taiwaneses overseas y los mainland. Previamente, es necesario dar cuenta de la historia de la migración hacia Argentina.

\section{Migración china y taiwanesa en argentina}

La primera corriente de inmigrantes chinos hacia Argentina, de acuerdo a Trejos y Chiang (2012) comienza durante la guerra entre China y Japón (1894) 
hasta 1949, cuando finaliza la guerra civil china. En este período, la inmigración no es importante en números, sino que se trata de hombres solos que llegaban con escasas sumas de dinero desde las zonas costeras de China o desde países como Perú y Chile. En 1914, según datos del INDEC, ${ }^{3}$ se registraron 463 personas de origen chino, de las cuales, el 60\% residía en la Ciudad de Buenos Aires y casi el 10\% en la Provincia de Buenos Aires (Bogado Bordázar, 2002).

Un segundo periodo de inmigración se abre desde 1949. Si bien aumenta el flujo de migrantes chinos, el número sigue siendo bajo. Las 100 familias que llegaron desde China estaban acompañadas por un sacerdote. Por los problemas que éste tuvo con el gobierno peronista, los migrantes decidieron seguir camino hacia países vecinos, fundamentalmente Brasil (Bogado Bordázar, 2002; Trejos; Chiang, 2012). En 1947 el INDEC estableció la presencia de 365 chinos mientras y según datos extraoficiales, el número ascendió en la década del 60 a 1500 .

Un tercer periodo comienza en los 70, con la llegada de chinos que en su mayoría escapaban al régimen comunista ${ }^{4}$ (Bretal, 2005; Sassone; Mera, 2007; Trejos; Chiang, 2012). El país recibe en los años 80 a numerosas familias taiwanesas. Entre las razones por las que deciden migrar, se establecen el miedo a la guerra o expansión de China sobre la isla de Taiwán, la búsqueda de una mejor calidad de vida, la ruptura con la tradición, ${ }^{5}$ o por razones políticas: ya sea el temor a que se desate una guerra con China o por persecución ideológica. ${ }^{6}$ La decisión de migrar es acompañada por un "consejo” que proviene de allegados a los que respetan. Pueden ser familiares o proveedores de sus negocios, pero varios informantes recalcan la idea de una persona prestigiosa, cuya opinión es tenida en cuenta por el padre de familia. ${ }^{7}$ Generalmente

3 Instituto Nacional de Estadísticas y Censos de Argentina.

4 Algunos informantes han indicado que estos primeros migrantes chinos eran en realidad taiwaneses, pero que en esas décadas aún no era clara la diferencia entre chinos y taiwaneses.

5 Algunos informantes mencionaron su disconformidad ante el rol que la tradición familiar china les asigna. Uno de ellos decidió migrar, en parte, porque no quería trabajar para mantener a sus hermanos, como se espera del hijo mayor. Otro decide buscar nuevos horizontes para así evitar casarse con la persona elegida por sus padres.

6 Diversos miembros de las iglesias presbiteranas manifestaron que la decisión de migrar surgió de saber que sus nombres figuraban en listas negras.

7 En muchos casos, los hijos no sabían las razones por las que su padre había decidido migrar. Las entrevistas con la investigadora fueron un puntapié para realizar la pregunta. También es frecuente encontrarse con familias que comentan que la decisión la tomó el padre, y la madre acompañó sin estar de acuerdo.

Horizontes Antropológicos, Porto Alegre, ano 21, n. 43, p. 79-103, jan./jun. 2015 
migran las familias enteras, quienes al llegar son ayudadas por parientes o amigos que ya se encuentran instalados en el país. En Taiwán dejan su profesión y su bienestar económico. La mayoría de los migrantes entrevistados argumenta haber tenido un buen pasar económico de la mano de sus profesiones que una vez que llegan a Argentina, deciden abandonar, fundamentalmente por las dificultades para homologar los títulos universitarios. A las cuestiones burocráticas se suman las vicisitudes con el lenguaje -ninguna familia sabía hablar español al llegar y muchos migrantes aún hoy siguen sin aprenderlo, pues sus hijos hacen de traductores cuando lo necesitan-, y las propias de las relaciones bilaterales entre Argentina y Taiwán. ${ }^{8}$ Estos migrantes contaban con dinero suficiente para montar sus negocios o comprar viviendas una vez instalados en Argentina (Bogado Bordázar, 2002). A mediados de los 90, la cantidad de taiwaneses ascendía a 25.000 personas, de las cuales 23.000 vivían en Buenos Aires y alrededores (Pappier, 2011).

En la misma década, se dio un importante flujo migratorio desde la RPC (Bogado Bordázar, 2002). Mayoritariamente llegaban sin capital aunque con grandes expectativas de progreso económico. Eligieron Argentina por considerarlo un país "seguro" y por la situación de crecimiento en la que se encontraba la economía local hasta 1997, que fundamentalmente les permitía ganar en dólares americanos. ${ }^{9}$ En esta década, fuentes extraoficiales afirmaban que existían entre 40.000 y 45.000 chinos en Argentina (Bogado Bordázar, 2002).

Finalmente, podríamos mencionar una cuarta ola migratoria. Desde 2001, la inmigración de China hacia Argentina ha variado en relación a las crisis y recuperaciones económicas de nuestro país. Al comienzo de la década, la población china ascendía a entre 30 y 50 mil habitantes (Zuzek, 2004). Luego de la crisis del año 2001, descendió a 20 mil y desde 2004 se incrementó nuevamente, coincidiendo o influenciado por la recuperación económica argentina. Según un informante perteneciente a la "comunidad", los taiwaneses hacen el proceso contrario que los chinos: luego de la crisis de 2001, los primeros emprenden viaje hacia Estados Unidos o Taiwán. Esta merma se explica en el trabajo de Bernardo Trejos y LanHung Nora Chiang (2012), por

8 Argentina no reconoce a Taiwán como país autónomo desde que entabló relaciones diplomáticas formales con RPC en 1979.

9 En esa década, el gobierno argentino había instaurado la Ley de Convertibilidad, por la cual un peso argentino era equivalente a un dólar norteamericano. 
las mejores oportunidades de trabajo que los jóvenes inmigrantes encuentran dado el boom de las economías de RPCh y Taiwán y el deseo de reunirse con sus familiares o la necesidad de cuidar de sus padres. Nuestros informantes afirman que la "inseguridad" y la "inestabilidad" en Argentina, produjo que muchos "paisanos” hayan tomando la decisión de irse del país. ${ }^{10}$

En 2007 fuentes extraoficiales calculaban en 120.000 la cifra de personas de origen chino en Argentina, distribuidos en las principales ciudades del país, fundamentalmente CABA y conurbano bonaerense, Córdoba, Mendoza, Tucumán, Mar del Plata, Rosario y La Plata ${ }^{11}$ (Zhang, T., 2007).

En la actualidad, nuestros informantes nos brindan las siguientes cifras: 12 mil taiwaneses, 200.000 chinos registrados y 100.000 más no registrados. ${ }^{12}$

Es decir, mientras que la inmigración china se produjo más recientemente y en mayor número, los taiwaneses llegaron en menor número hace más de 40 años, y alcanzan el 20\% de la población china. Esto se traduce en una diferencia significativa en términos de aprendizaje de idioma e integración a la sociedad de residencia.

Brage (2010, p. 7) afirma que esta diferencia es notable incluso al interior de la comunidad taiwanesa "en el desempeño de la lengua española y los usos aprendidos en nuestra sociedad así como también en el grado de integración”. Las generaciones más adultas no han aprendido a hablar bien español, a pesar de los años de permanencia en Argentina. Esto es parte se debe a que en

10 Algunos supermercados propiedad de migrantes chinos fueron saqueados. Constantemente, los “supermercados chinos” son noticia por haber sufrido asaltos, algunos muy violentos.

11 Alejandra Pappier (2011) hace alusión a una pequeña cantidad de migrantes en Jujuy, Chaco y Santa Cruz.

${ }^{12}$ Los datos del Censo de Población, Hogares y Viviendas de 2010, arrojan una cantidad de casi 9 mil personas que nacieron en la RPCh y casi 3 mil que nacieron en Taiwán, dentro del territorio argentino (http://200.51.91.245/argbin/RpWebEngine.exe/PortalAction?\&MODE=MAIN\&BASE=CPV2010B\& MAIN=WebServerMain.inl). Los datos estadísticos correspondientes a la población china y taiwanesa en Argentina revisten al menos tres grandes problemáticas. En primer lugar, muchos migrantes más allá de la situación de ilegalidad o no, no responden a los censistas, por lo que las cifras oficiales difieren marcadamente de las no oficiales. En segundo lugar, en los datos, gráficos y tablas no hay discriminación entre China y Taiwán, otra consecuencia de la política argentina de no reconocer a Taiwán como país independiente. Finalmente, existen escasos registros de los primeros flujos de migración, su composición y densidad (Casals, 2009). Estos inconvenientes, sumado a la desactualización de los datos estadísticos frente a una realidad que se modifica a gran velocidad, y el interés de las organizaciones por presentar una población mayor fines políticos, generan una diferencia importante entre los datos oficiales provenientes de INDEC o de la Dirección de Migraciones, y los que pueden brindar la Embajada de RPC, la Oficina Comercial de Taiwán o las grandes Cámaras que nuclean a los migrantes

Horizontes Antropológicos, Porto Alegre, ano 21, n. 43, p. 79-103, jan./jun. 2015 
las asociaciones e instituciones se habla chino mandarín o dialecto taiwanés. Una vez que sus hijos comenzaron con la escolaridad y aprendieron el idioma, oficiaron de traductores de sus padres. Encontramos entonces que los mayores de 60 años escasamente hablan español, mientras que los migrantes o hijos de migrantes de entre 30 y 40 años hablan fluidamente español y mandarín. Algunos de ellos incluso manejan el dialecto taiwanés, muy utilizado en la cotidianeidad del hogar.

En este grupo etario estamos observando una revalorización económica de un rasgo anteriormente discriminador. Mientras que a edades tempranas los migrantes e hijos de migrantes sufren las burlas de sus pares por sus rasgos fenotípicos, lo que implica su rechazo a hablar en chino, actualmente los jóvenes bilingües están siendo muy solicitados como traductores e intérpretes por empresas multinacionales o nacionales que quieren abrir mercados en China, o empresas chinas que se radican en Argentina. Los hijos de migrantes que nacieron en Argentina y que actualmente tienen 25 años o menos, manejan menos fluidamente el mandarín y escasamente el dialecto.

La actividad laboral que desarrollan los migrantes no ha variado a lo largo de estos años. Los taiwaneses que llegaron en los 70 y 80 instalaron almacenes, o supermercados, restaurantes, lavaderos de ropa y casas de revelado de fotos, rubros en los que no hace falta conocer demasiado el idioma del lugar de residencia. ${ }^{13}$

Al llegar a Argentina, las familias taiwanesas en su mayoría eligieron barrios de clase media, o media alta para comprar una vivienda. También en su mayoría envían a sus hijos a escuelas privadas, sin importar si pertenecen a la religión católica. Prefieren una escuela exigente y católica, y no una pública donde consideran que se imparte una educación de baja calidad. Además de asistir al "colegio argentino", los fines de semana asisten al "colegio chino", ${ }^{14}$ para aprender chino mandarín, instrumentos musicales chinos, artes marciales,

13 La literatura al respecto de los nichos laborales de los migrantes chinos en otros países, nos hace pensar que además de la cuestión idiomática, existe una tendencia a repetir actividades desarrolladas por migrantes en otros destinos. Fleischer (2012) indica que la legislación de Estados Unidos durante la recesión económica de 1930, mantenía a los chinos por fuera de muchas profesiones. Las lavanderías y las tiendas de provisión, “considerados trabajos de mujeres, no eran considerados importantes como para restringir el ingreso de los chinos” (Fleischer, 2012, p. 75).

${ }^{14}$ Los “colegios chinos” de Buenos Aires son gerenciados por taiwaneses y quienes egresen de estas instituciones, ingresan sin necesidad de rendir examen a las universidades taiwanesas. Reciben gran cantidad de niños de familias chinas.

Horizontes Antropológicos, Porto Alegre, ano 21, n. 43, p. 79-103, jan./jun. 2015 
entre otras artes. Los compañeros de colegio chino será con posterioridad un grupo de referencia importante en la vida de los informantes. Algunos nos mencionaron que los padres taiwaneses también inculcan la pasión por algún instrumento musical o un deporte y el aprendizaje de inglés. Se hace hincapié para que los hijos tengan un título universitario, lo cual entra en tensión con la "raíz comerciante de la cultura china". Es frecuente que los informantes afirmen dejar que las hijas mujeres estudien, mientras que los hijos varones se dediquen a continuar el negocio familiar o abran el propio.

La migración china más reciente llega desde zonas empobrecidas de China (Fujian, principalmente), sin capital económico y con menor nivel de instrucción -muchos de los migrantes no hablan mandarín sino dialecto y no tienen estudios universitarios-. La edad promedio de los migrantes es muy baja, alrededor de los 25 años. ${ }^{15} \mathrm{Al}$ llegar, compran un fondo de comercio de un supermercado, deuda que se suma a la que contrajeron para viajar e ingresar al país. ${ }^{16}$ Es por eso que circula extendidamente entre los argentinos la alusión a que "viven para trabajar", ya que los primeros años de residencia en el país, deben saldar las deudas contraídas. Las jornadas laborales tan extensas hacen que sean escasas las asociaciones culturales chinas. La mayoría de los chinos reside en el mismo lugar que trabaja. Los supermercados cuentan con habitaciones anexas o en pisos superiores donde duerme toda la familia. Con el tiempo, algunos están en condiciones de alquilar un lugar más acogedor.

En la Ciudad de Buenos Aires encontramos un supermercado propiedad de migrantes chinos cada dos o tres cuadras. Esta saturación de la ciudad los llevó a expandirse a las segundas ciudades del país, como Rosario, Córdoba y Mar del Plata. Desde la Federación de Supermercadistas Chinos, se estima que en Argentina existen actualmente 8.900 supermercados, de los cuales 3.500 se encuentran en el Gran Buenos Aires y la Ciudad Autónoma. Otros negocios desarrollados por estos migrantes, son los "bazares” o "regalerías” que en un mismo local ofrecen a la venta artículos de decoración, para el hogar, vestimenta y juguetes importados chinos.

15 Observamos que muchos migrantes son jóvenes que comienzan a formar su familia. Algunas veces los niños son enviados a China para ser educados por los abuelos, en los valores y en el idioma chinos. De esta manera, los padres disponen de más tiempo para trabajar.

16 Algunos informantes nos han indicado que el dinero se reúne entre los vecinos de aquellos que quieren migrar. Es una deuda que se debe saldar, sin necesidad de extender ningún tipo de garantía. 
Últimamente están llegando al país jóvenes chinos motivados por aprender el español, que se desempeñan como profesores de idioma chino para argentinos y realizan estudios de posgrado en las universidades locales. También es más frecuente la llegada de empleados de las empresas chinas que comienzan a radicarse en el país (SINOPEC, AXION ENERGY), que revisten grandes diferencias con sus anteriores compatriotas, en términos educativos, económicos y de clase.

\section{Sentidos de ser chino en Buenos Aires}

Es imprescindible, para la completa comprensión de este proyecto, introducir al lector en una serie de cuestiones históricas y políticas que determinan las relaciones y las identificaciones entre los inmigrantes chinos y taiwaneses.

Como adelantamos, en 1912 un grupo de estudiantes chinos que se encontraban en Japón, en medio del descontento por la pérdida de la guerra sino-japonesa, proclamó la República de China y la abdicación del último emperador de la Dinastía Qing (Gelber, 2008, p. 249). Los años que siguieron fueron de una profunda confusión política. Japón, aprovechando la primer guerra europea, y sintiéndose obligado por la fraternidad étnica a ayudar a China en el camino a la modernización, en 1915 ya había consolidado el dominio en zonas como Taiwán, Corea, Manchuria y Mongolia, logrando que China aceptase consejeros japoneses económicos y militares, convirtiéndose en un protectorado japonés (Gelber, 2008, p. 256).

Al finalizar la guerra, los resultados del Tratado de Versalles favorables a Japón, iniciaron un movimiento marxista anti imperialista y nacionalista, que recibió apoyo de Moscú, marcando el comienzo de una intensa relación entre China y la ex URSS. En 1925 muere Sun Yat-sen, el primer presidente de la República de China (Taiwán) y fundador de Kuomintang (Partido nacionalista chino). Lo sucedió Chiang Kai-shek quien rompió relaciones no sólo con los soviéticos sino con el Partido Comunista Chino. La persecución a militantes del partido terminó en una guerra civil. Los comunistas iniciaron entre la década del 30 y el 40 una guerra de dos frentes: los nacionalistas y los japoneses. Lentamente fueron contando con el apoyo de los campesinos víctimas de actos de violencia y corrupción por parte de estos últimos.

Con la rendición de Japón luego de las bombas de Hiroshima y Nagasaki en la segunda guerra mundial, también se terminó la ocupación japonesa en 
China. La guerra civil entre el Kuomintang y los Comunistas continuaría hasta 1949, cuando los nacionalistas, derrotados escaparon hacia Taiwán.

Las fronteras de China quedan cerradas y se inicia el periodo Comunista bajo las órdenes de Mao Tsé Tung. En Taiwán, la complicidad entre el Kuomintang y Estados Unidos derivó en la implantación del modelo capitalista. A la violencia que trajeron las invasiones japonesas, le siguió la de los nacionalistas, quienes impusieron un gobierno dictatorial que diezmó numerosas etnias taiwanesas, y cercenó libertades de los pobladores, por ejemplo, prohibiendo el uso de dialectos.

Actualmente, la República Popular China (RPCh) no acepta la independencia de Taiwán (República de China), sino que la considera una provincia más con un régimen político y económico diferente. ${ }^{17}$ Taiwán se autoproclama provincia autónoma en la que rige un sistema político democrático, con pluralismo partidario. Se produce también una ruptura a nivel lingüístico ya que en China continental, comienzan a utilizar el "chino simplificado” y en Taiwán continúan hablando el chino mandarín "tradicional”. ${ }^{18}$

La historia de China y Taiwán es desde entonces, una sucesión de acercamientos y distanciamientos, de intentos de unificación de un lado y de reclamos por la autonomía e independencia por el otro. Recién en el año 2000 cuando fue elegido el primer presidente democrático de Taiwán, cesó el estado de guerra entre los dos gobiernos. Sin embargo, como hemos analizado en trabajos anteriores (Denardi, 2013) la situación política no deja de estar en el centro de la escena en los grupos de ultramar en Buenos Aires. Sea por intentos de omisión, de fusión, o de diferenciación, "la cuestión Taiwán” se cuela en los repertorios de los inmigrantes.

\section{Ser taiwanés y ser chino "en sentido amplio"}

Durante el trabajo de campo, algunos migrantes taiwaneses se identifican como chinos, otros como taiwaneses y otros reacomodan su identidad dependiendo de las diferentes situaciones que se les presentan.

${ }^{17}$ Los chinos continentales utilizan la frase "Un país, dos sistemas” para referirse a esta cuestión. Para ampliar este tema Ver: Pinheiro Machado (2010).

${ }^{18}$ El chino simplificado formó parte de una política para aumentar la tasa de alfabetización a través de la simplificación -escritura con menos trazos- del chino mandarín. Actualmente, existen claras diferencias de acentuación y vocabulario entre la RPCh y Taiwán. 
Si me encuentro frente a una china, voy a decir que soy taiwanesa. Pero si vos [argentina] me preguntás, mis antepasados vienen de China, así que sí, soy china.

Sugerimos entonces, que surgen al menos dos sentidos de ser "chino" en Buenos Aires: Por un lado, ser chino en sentido estrecho es decir, el inmigrante que proviene de zonas donde se reconoce la jurisdicción de la RPC. Por otro lado, podríamos hablar de ser chino en sentido amplio. ${ }^{19}$ aquellos que por sus costumbres y antepasados, se sienten pertenecientes a la cultura china, que excede a la cultura de habitantes la República Popular de China.

En el caso de los taiwaneses, estas identificaciones implican posiciones morales respecto a los chinos. La "etnografía de las moralidades" (Fassin, 2008; Howell, 1997) implica interrogarse acerca de los conflictos y los consensos, los acuerdos y los desacuerdos respecto de la construcción, mantenimiento, impugnación y negociación de diversas configuraciones de valores sostenidos como legítimos o deseables por determinados actores sociales. Siguiendo a Archetti (2003), entendemos las moralidades no sólo como la evaluación sobre lo correcto y lo incorrecto, lo bueno y lo malo, sino también sobre lo deseable y lo indeseable, lo meritorio y lo no meritorio para una comunidad.

Indagaremos entonces, las diferentes posiciones morales que reconocemos entre los taiwaneses en relación a la mayor o menor cercanía con los chinos en sentido estrecho.

En primer lugar, como ya adelantamos, existe una clara diferencia generacional entre las diferentes olas migratorias. La primera gran ola de taiwaneses, llegó durante los años 80, cuando aún no se habían producido las consecuencias de las políticas de apertura en China, y se mantenía gran tensión política con Taiwán. La brecha que separaba un Taiwán industrializado, con casi nulos niveles de analfabetismo, con una China empobrecida e inculta, era mayor que en la actualidad. La cultura taiwanesa se presenta como superior a la de China Continental, porque la consideran "pura" al no haber sufrido la destrucción de la Revolución Cultural y la simplificación del idioma. Al referirse a los

19 Utilizando las mismas palabras de la definición de Edel y Edel (1959) sobre la ética: “ethics wide” y “ethics narrow”. El sentido estrecho sería el inmigrante chino que proviene de China Continental. 
chinos en sentido estrecho, los informantes taiwaneses de esta generación nos explican que

hay una cuestión de educación. Con la Revolución Cultural se destruyó mucho, se hizo mucho desastre, y Taiwán trató de conservar la cultura, el idioma. Los chinos que van a Taiwán se asombran cuando los taiwaneses dicen perdón, gracias, por favor. Los chinos no dicen nada de eso.

Durante dos décadas, en Buenos Aires el grupo de los taiwaneses era numéricamente mayor al de los chinos, por lo que se consideraban los únicos chinos del país. Cuando llegaron los chinos en sentido estrecho, los taiwaneses comenzaron a marcar fronteras entre los grupos. Algunas organizaciones sólo aceptan miembros con pasaporte taiwanés, también encontramos iglesias que realizan sus cultos en dialecto de Min Nan, más conocido como dialecto taiwanés, el cual excluye a migrantes chinos; y las celebraciones culturales más importantes se realizan paralelamente a otras que incluyen chinos.

Pero estas fronteras no sólo se trazan físicamente, sino que también son de tipo moral. En palabras y gestos de los adultos mayores que conforman la primera generación de taiwaneses. Los chinos son, a los ojos de este grupo de taiwaneses, poco confiables.

Los chinos creen que somos todo lo mismo, y no es así. El chino es tramposo. Ellos te dicen "hacé, hacé" y si sale algo mal "ellos no fueron" -levantando las manos como mostrando desentendimiento-.

Y otra cuestión que parece diferenciarlos es la "humanidad":

Taiwán ayudó a China en el terremoto de Sichuan y cuando hubo un terremoto en Taiwán ellos no ayudaron. Taiwán donó mucho dinero a Japón con el tsunami, China también, pero ellos son muchos, tendrían que haber donado mucho más. Esto te muestra que la humanidad y el amor están en Taiwán.

En palabras de estos informantes, los "chinos" en sentido estricto serían personas de humanidad degradada, ${ }^{20}$ ya que no son ni tan cultos, ni tan

20 "El régimen de deshumanización se caracteriza por una metafísica [...] orientadora de una gramática de justificación pública que busca extraer ciertos seres de una humanidad común” (Freire, 2010, p. 120, mi traducción). 
solidarios, ni tan responsables como los taiwaneses. Como afirma Jussara Freire (2010), este repertorio moral de deshumanización o de humanización degradada, indica la sensación de no reconocimiento como portadores del capital cultural que detentan.

La distancia moral y física que marcan los taiwaneses de la primera generación con los chinos, no es la misma que surge entre los de la "generación $1.5^{21}$ y de los hijos de taiwaneses nacidos en Argentina. Algunos informantes de esta generación iniciaron dos procesos en los que los miembros de la primera generación no participan: comenzaron a negociar con los chinos y a realizar eventos conjuntamente con ellos.

Rosa $^{22}$ es una mujer taiwanesa de aproximadamente 70 años que promovió acciones para que el Barrio Chino $^{23}$ sea incluido en el circuito turístico de la Ciudad y financia obras culturales de miembros de la "comunidad", cuestiones que la convierten para muchos informantes, en una referente y benefactora. Otros "paisanos"24 impugnan sus actos por la supuesta riqueza que ostenta:

D:- treinta años hace que la conozco [a Rosa]. No tenemos lo mismo códigos (sic).

L:- cuáles son esos códigos?

D:- es millonaria, a mi no me importa la plata, no me importa tener cosas caras.

Es interesante indicar que para los chinos y taiwaneses, la fortuna no es un valor despreciable, sino que por el contrario, se lo incluye en los rezos a Buda, se desea buena fortuna a familiares y conocidos en Año Nuevo, el ideograma que lo representa puede ser encontrado hasta en los lugares más recónditos, pequeños sobres rojos con dinero son entregados en diversas ocasiones. Es decir, la impugnación a Rosa no proviene de su fortuna, sino de la manera en que la logró: negociando con los chinos en sentido estrecho.

${ }^{21}$ Los taiwaneses se autodenominan y autoidentifican como generación 1.5 para dar cuenta de que nacieron en Taiwán y llegaron a Argentina con 10 o 12 años.

${ }^{22}$ Los nombres de nuestros informantes han sido modificados a los fines de mantener la confidencialidad de sus palabras.

${ }^{23}$ El Barrio Chino de la Ciudad de Buenos Aires. Consta de aproximadamente 6 cuadras en las que se intercalan supermercados de productos asiáticos, regalerías de artículos importados chinos, casas de te, y restaurantes chinos, japoneses y vietnamitas. Las asociaciones taiwanesas más antiguas y una iglesia budista china tienen sus sedes allí.

${ }^{24}$ De esta manera los taiwaneses denominan a aquellos migrantes que provienen de la misma tierra. 
Los sujetos diaspóricos no son considerados sujetos de derecho ni por el Estado de lugar de origen, ni por el Estado de la sociedad de recepción, ya que no son el objetivo de las políticas públicas. Es por esto que la diáspora debe generar sus propios procesos de producción del bienestar. ${ }^{25}$ Rosa inició procesos que generan bienestar en la diáspora, como la cobertura médica para los migrantes chinos, la posibilidad de viajar a China a precios más bajos a través de su agencia de viajes y los mayores ingresos que obtuvieron los comerciantes a medida que el barrio se fue constituyendo como un punto turístico fuerte de la ciudad. ${ }^{26}$ Estas serían las maneras en las que Rosa consiguió acumular una buena fortuna.

Un segundo punto a destacar, consiste en analizar que las causas de impugnación moral no están relacionadas con valores que podemos catalogar como "chinos", sino con repertorios "argentinos". La riqueza de esta mujer se mide en términos "argentinos”, haciendo alusión a la zona en donde está emplazada su propiedad. Esto nos indicaría que también la manera en que construimos los repertorios morales (Noel, 2013) y los valores que utilizamos para evaluar las prácticas propias y ajenas, se transforman durante el proceso migratorio.

Un tercer punto es la tarea casi pedagógica que realizó Rosa: algunos taiwaneses, que se consideran a sí mismos chinos en sentido amplio, aprendieron de ella ciertas “justificaciones”, entendidas por Werneck (2013, p. 709, mi traducción) como "dispositivos de respuesta a juzgamientos morales [que] surgen mediante críticas o acusaciones.”

Entonces en el 2006 quería armar y justo me vino a hablar Rosa, "che tenemos que hacer algo" [me dijo], y entre nosotros dos lo armamos [el festejo del Año Nuevo].

L:- ¿vos ya la conocías [a Rosa]?

A:- si, la conocía. Yo no la quería, pero tenía el objetivo de armar eso [el festejo del Año Nuevo]. Porque yo sé que solo no puedo.

L:- ¿porque no la querías?

A:- porque había comentarios de eso que se acerca con los chinos, hace negocios con los chinos, yo soy muy taiwanés viste.

${ }^{25}$ Agradezco los comentarios de Federico Besserer y Mónica Cinco al respecto.

${ }^{26}$ Actualmente, se calcula que el Barrio Chino de Buenos Aires recibe a 20.000 personas los fines de semana. Durante las fiestas como el Año Nuevo, la cantidad de asistentes asciende a 120.000. 
Frente a la acusación de que "negociar con chinos" no es un signo de lealtad hacia Taiwán, la manera de acercarse a los chinos en sentido estrecho sin la condena moral implica mantener y defender la soberanía taiwanesa. Nuestro informante afirma que fue Rosa la que le proveyó de una justificación:

Trabajando con ella me abrió mucho la mente, ${ }^{27}$ o sea, todos son chinos, ¿̇porqué tenés que dividir [en] taiwanés [y] chino? Cuando te toca lo que es soberanía, si defendéte, pero si es una cultura, es lo mismo.

Es así como nuestro informante logró mantener un equilibrio: negocia con los chinos, a la vez que mantiene su identidad y moralidad taiwanesa. Participa de las asociaciones taiwanesas a la vez que trata de eludir las afirmaciones políticas sobre las relaciones China-Taiwán. Para los festejos del Año Nuevo Chino, logró que no hubiese banderas, ya que según él, es una forma de mantener intacta la soberanía.

La tercera posición es la encarnada por algunos taiwaneses que se consideran chinos “en sentido amplio”, y que al no tener una justificación legítima para quienes se ubican en las posiciones anteriores, son considerados "traidores" que se "venden a los chinos" y no reinvindican su identidad taiwanesa. Estas personas intentan eliminar las diferencias al interior de la diáspora (Louie, 2000, p. 651) a través de la inclusión de taiwaneses, chinos en sentido estrecho y chinos en sentido amplio en el legado ancestral chino, en la "cultura china” que tiene 5000 años de historia, muchos más que los 60 años de conflicto entre China y Taiwán.

\section{Ser chino en sentido estrecho}

Dentro de la diáspora china en sentido estrecho, podemos identificar 3 grupos de migrantes. Por un lado, los supermercadistas que ya suman entre 10 y 20 años en el país. En segundo lugar, los miembros jerárquicos de las "asociaciones de residentes" chinos en Argentina y por último, la mano de obra calificada que llega para ocupar puestos dirigenciales en las empresas chinas que están instalándose en nuestro país.

${ }^{27}$ Forma coloquial de expresar reflexión, salir de las estructuras propias de pensamiento e incorporar nuevas ideas. 


\section{Supermercadistas chinos}

Durante la década de los 90, muchos migrantes chinos llegaron a Argentina gracias a los "llamados" que realizaban los que ya estaban residiendo en nuestro país, de sus parientes que aún residían en China o Taiwán. En esta década, proliferaron los restaurantes "tenedor libre", ${ }^{28}$ propiedad de migrantes chinos. Alrededor de 1995 comienzan a abrirse los primeros supermercados, que a partir del año 2004, con el incremento de la migración, cobraron notoriedad por la gran cantidad de locales y los precios bajos y largas jornadas de trabajo que incluían sábados, domingos y feriados. ${ }^{29}$ Esta actividad es el principal medio de inserción económica de los migrantes y tuvo dos consecuencias fundamentales en la organización de la comunidad china en Argentina. Por un lado, generó una dispersión urbana de la "comunidad". Actualmente encontramos supermercados cuyos dueños son de origen chino cada 3 cuadras en todos los barrios de la CABA, extendiéndose a las principales ciudades argentinas. En segundo lugar, y en parte como consecuencia de lo primero, la mayoría de los migrantes no vive en el Barrio Chino, sino en las zonas donde instalan sus comercios.

Los supermercados y restaurantes propiedad de migrantes chinos han sufrido diversos embates discriminatorios y delictivos. Mediáticamente, se reproduce el prejuicio de que apagan las heladeras de los lácteos durante la noche para ahorrar energía, que cocinan ratas, que son sucios, que almacenan alimentos frescos junto a la basura, etc. ${ }^{30}$ Recién a fines de 2013 desde la comunidad taiwanesa, dos voceros tuvieron derecho a réplica en los medios locales. Desde la Asociación Barrio Chino se está trabajando para "educar” al comerciante. Reconocen que en algunos locales de venta de comida

${ }^{28}$ Se conocen popularmente como "tenedor libre" aquellos restaurantes que pagando un precio fijo, el cliente puede servirse cuantas veces quiera, gran variedad de comidas.

${ }^{29}$ En casi todos los comercios chinos, la estrategia de venta consiste en bajar los precios para lograr una gran salida de productos. Poco a poco, los supermercadistas chinos fueron nucleándose en Federaciones que lograban bajar aún más los precios al comprar en grandes cantidades, para abastecer a todos los supermercados.

${ }^{30}$ Existe una asociación de vecinos del Barrio de Belgrano que se dedica a denunciar, sacar fotos y publicar en internet estas situaciones, de la que los medios se hacen eco. En este blog se denuncia además, un encubrimiento del gobierno de la Ciudad (http://belgranodenuncia.blogspot.com.ar/). Como consecuencia, las ventas y la cantidad de personas que concurren a los restaurantes del Barrio Chino merman considerablemente. 
a la calle en el Barrio Chino se cocina en el piso, como los migrantes están acostumbrados a hacer en sus lugares de origen. Su tarea entonces implica enseñar a estos comerciantes que las leyes argentinas no permiten esa manera de cocinar. ${ }^{31}$

\section{Asociaciones de residentes}

Los documentos oficiales del Gobierno Chino, principalmente el renombrado Libro Blanco ${ }^{32}$ (Moneta; Cesarín, 2012), en relación a las expectativas en América Latina, hablan del beneficio mutuo, de la necesidad de mantener relaciones emotivas, afectivas, para el posterior desarrollo de relaciones económicas o materiales. El establecimiento de guanxi, ${ }^{33}$ tanto en las relaciones empresariales como en las políticas y económicas a nivel gubernamental es considerado una de las reglas principales para el éxito.

Las empresas transnacionales asiáticas y fundamentalmente las inversiones provenientes de China, no sólo vienen a América Latina en busca de materias primas e insumos sino que además, tienen como objetivo ampliar los mercados en el exterior para hacer de sus firmas locales marcas globales (Moneta; Cesarín, 2012).

En Argentina los lazos diplomáticos con China han sido establecidos fundamentalmente sobre argumentos económicos, manteniendo una relación pacífica bilateral desde hace casi 40 años (Zhao, 2012 apud Moneta; Cesarín, 2012, p. 103). Las principales inversiones se realizan en sectores como el transporte público, pescadería, frigoríficos, agricultura, ensamblaje de automotores y productos electrónicos, telefonía, obras civiles y de infraestructura, minería, petróleo y gas, fertilizantes, y fabricación de electrodomésticos.

${ }^{31}$ Algunas notas que hemos publicado al respecto: Denardi (2013b), Ng y Denardi (2014).

${ }^{32}$ China's policy paper on Latin America and the Caribbean (2008).

${ }^{33}$ Guanxi es la categoría que hace alusión a la capacidad de crear fuertes y amplias relaciones interpersonales basadas en el interés y el beneficio mutuo (Ordóñez de Pablos, 2004, p. 222). Literalmente tiene dos significados: conectar, hacer un vínculo y pasar por una puerta. Hace alusión a la habilidad de hacer conexiones sociales para negociar pases por puertas a lo largo de su camino. Actualmente, es una de las categorías utilizadas por diversos autores para explicar el éxito de los emprendimientos comerciales tanto de los chinos de ultramar, como de la República Popular China. Sin embargo, el guanxi no se limita a entablar relaciones con fines comerciales, sino a cualquier tipo de relación, que implica "el intercambio de regalos, favores y banquetes; el cultivo de relaciones personales y redes de mutua dependencia; y la creación de obligaciones y deudas” (Yang, 1994, p. 6, mi traducción). 
Este auge económico ha sido acompañado por una renovación del lazo entre el Estado Nación y sus diásporas. Anteriormente, China no reconocía a la población de ultramar; en la actualidad comenzó a integrarla en el entramado transnacional de su política económica. Guerra Zamponi (2010) menciona que la actual política china sobre sus migrantes de ultramar los insta a mantener vínculos transnacionales desde los lugares de residencia con la madre patria. ${ }^{34}$ Las "asociaciones de residentes" en Argentina, son uno de los pilares que más se ha desarrollado actualmente. ${ }^{35}$

Estas asociaciones revisten formas muy heterogéneas de funcionamiento. Principalmente, reúnen migrantes provenientes de una determinada ciudad o región. Se trata de hombres de negocios, que comenzaron como supermercadistas y que fueron escalando posiciones hasta dedicarse a la importación y exportación de productos entre China y Argentina. En la actualidad hemos relevado más de 20 organizaciones de este tipo en Buenos Aires. ${ }^{36}$ Generalmente, tienen escasa visibilidad incluso para los miembros de la misma diáspora. Muchas de ellas no tienen sede propia, y utilizan restaurantes para reunirse con mayor o menor periodicidad (Grimson; Ng; Denardi, 2014).

Fundamentalmente, lo que nos interesa recalcar, es cierta imposibilidad de diferenciar entre actividades formales e informales, privadas o públicas, característica propia de las diásporas en contexto transnacional (Barabantseva, 2012, p. 91). Estas asociaciones mantienen fuertes lazos con la embajada china en Argentina y reciben funcionarios y empresarios chinos para quienes brindan grandes banquetes (Grimson; Ng; Denardi, 2014).

Los objetivos de las "asociaciones de residentes" son principalmente económicos: unir a los residentes chinos de determinada ciudad o región,

${ }^{34}$ El artículo de Guerra Zamponi (2010) se basa en las experiencias de Canadá y Australia, en las que estos mecanismos están en funcionamiento desde hace más tiempo, ya que el proceso migratorio hacia esos países es mayor y se inició tempranamente, en relación a la migración hacia Argentina. La distancia geográfica y por ende, los costos de los viajes entre China y Argentina, también son datos importantes que limitan la cantidad de viajes hacia el lugar de origen, en comparación con países como los mencionados o Estados Unidos.

${ }^{35}$ Si bien esfuerzos de este tipo a nivel privado se están realizando desde hace 30 años, mediante los vínculos creados por Cámaras de empresarios argentinos que vinculan empresas locales con pares chinas, la iniciativa estatal china surgió desde hace relativamente pocos años.

${ }^{36}$ Realizamos los contactos para la investigación dirigida por Alejandro Portes (Universidad de Princeton), denominada “Migraciones asiáticas en América Latina y sus relaciones transnacionales”. La investigación desarrollada en Buenos Aires fue dirigida por el Dr. Alejandro Grimson.

Horizontes Antropológicos, Porto Alegre, ano 21, n. 43, p. 79-103, jan./jun. 2015 
para generar acuerdos y negocios entre la ciudad que representan y Argentina. Secundariamente, se proponen actividades de integración, como la celebración de fiestas chinas y la apertura de escuelas bilingües (Grimson; Ng; Denardi, 2014).

Es decir, las asociaciones de residentes chinos son una esfera que se ubica entre el país de acogida y la madre patria, ubicación que las transforma en nodos de las redes que podrían realizar acciones efectivas en pro del intercambio entre Argentina y China. Con el objetivo de participar en el desarrollo económico de China, los migrantes de ultramar tienen un trabajo específico que es servir de red para generar vínculos comerciales entre los lugares de residencia y de origen, como sucede en Canadá o Australia (Guerra Zamponi, 2010).

\section{Clase alta empresaria}

La llegada de empresas chinas a Argentina y la creciente cantidad de convenios empresariales entre ambos países, ${ }^{37}$ viene acompañada de una migración muy diferente a la de los 90 y 2000. Se trata de alrededor de 3000 empresarios y profesionales altamente capacitados, que intercalan 3 meses de residencia y trabajo en Argentina y un mes de vacaciones en China, frecuentemente con escala en Europa. Otros llegan a hacer capacitaciones o asesorías solo por 6 meses. La mayoría indica que estos movimientos no son predecibles y que dependen de sus superiores, por lo que no conocen su próximo destino, o la duración exacta de su estadía.

Viven en edificios de alta categoría en uno de los barrios más caros de Buenos Aires, cercanos a las oficinas de sus empresas. Toman vacaciones en Europa, visten ropa cara y a la moda y asisten a restaurantes chinos exclusivos. Algunos de estos empresarios que conocimos se formaron en Europa, tienen más de un título universitario y hablan español e inglés.

Si bien hace tiempo que estos empresarios están trabajando en nuestro país, la prensa local los identificó hace pocos meses, titulando una nota periodística con las categorías "invasión silenciosa” y los "golden boys chinos” (Torino, 2014). La nota no hace más que poner de manifiesto una impugnación moral que recorre el sentido común de gran parte de los argentinos,

${ }^{37}$ Durante la visita del presidente Xi Jinping a la Argentina, en julio de 2014, se firmaron alrededor de 200 acuerdos comerciales entre empresas chinas y argentinas. 
para quien ser chino y adinerado y profesional es un oxímoron. Sin embargo, no dudamos que entre los sectores empresariales argentinos, la llegada de la economía china al puesto número uno del mundo, ${ }^{38}$ la imponente visita del presidente Xi y una comitiva de más de 200 empresarios, las cifras de las inversiones chinas en Argentina, ${ }^{39}$ se está posicionando a los migrantes chinos en otro lugar. Así, el migrante chino deja poco a poco de ser "el que apaga las heladeras de los lácteos”, para convertirse en la persona clave para una inserción económica exitosa.

\section{Conclusiones}

En este artículo expusimos algunas particularidades de la diáspora china en Argentina. A diferencia de algunos países en los que chinos y taiwaneses viven en ciudades separadas (Waldinger; Tseng, 1992), en Buenos Aires conviven de manera pacífica pero no sin mostrar algunas diferencias. En la “comunidad” china y taiwanesa se están generando conflictos y separaciones, invisibilizados en las cuadras del Barrio Chino y en los festejos y eventos culturales.

El nuevo lugar que toma China en la economía mundial ha generado diferentes consecuencias en la comunidad taiwanesa. Por un lado, las primeras generaciones, acostumbradas a ser los únicos chinos (en sentido amplio) del país, se sintieron invadidos cuando se modificaron las políticas chinas y permitieron que una gran cantidad de migrantes chinos "en sentido estrecho" llegara al país. De esta forma, y en segundo lugar, los taiwaneses comenzaron a impugnar a aquellos "paisanos" que se relacionaban con chinos por cuestiones económicas o culturales. Así, surgieron diferentes posiciones y justificaciones morales para lograr un equilibrio entre ser un buen taiwanés que no traiciona la causa de la autonomía de Taiwán, y generar beneficios económicos de la mano de los chinos, aprovechando una coyuntura global. Las evaluaciones morales realizadas por los taiwaneses sobre los "paisanos" se nutren de acusaciones cuyos sentidos solo pueden comprenderse en Argentina, por lo que nos invita a pensar que los repertorios morales se transforman en el proceso

${ }^{38}$ Aunque ellos renieguen de esto. Ver Giraldo (2014).

${ }^{39}$ Se realizaron anuncios de inversión por 6800 millones de dólares y salvataje financiero de 13 mil millones de dólares. 
migratorio, sumando a los adquiridos en el lugar de origen, aquellos propios del lugar de recepción.

En tercer lugar, los jóvenes taiwaneses se desempeñan como traductores e intérpretes en eventos económicos y son altamente remunerados en empresas locales interesadas en capacitarse o negociar con chinos. También como profesores de la lengua a los argentinos. Esto genera una revalorización de cualidades que antes eran objeto de estigma.

Finalmente, entendemos que la diáspora china funciona como un "puente" que une la sociedad de destino con la sociedad de recepción. Las "asociaciones de residentes" están insertas en un plan económico tendiente a la expansión de China hacia Argentina y América Latina. Prueba de esto es que la mayoría de las actividades de estas asociaciones implican la recepción de empresarios y funcionarios chinos en Argentina, y no están interesados -aúnen llevar a empresas argentinas a China.

De esta forma, esperamos haber colaborado, a través del conocimiento de las lógicas y divisiones de la diáspora china (en sentido amplio y en sentido estrecho), al entendimiento de las dinámicas de expansión económica global de la RPC.

\section{Referencias}

ARCHETTI, E. Masculinidades: futbol, tango y polo en la Argentina. Buenos Aires: Antropofagia, 2003.

BARABANTSEVA, E. Who are "overseas chinese ethnic minorities"? China's search for transnational ethnic unity. Modern China, v. 38, n. 1, p. 78-109, 2012.

BOGADO BORDAZAR, L. Migraciones internacionales. Influencia de la migración china en el Río de la Plata. 2002. Tesis (Maestría en Relaciones Internacionales)-Universidad Nacional de La Plata, 2002.

BRAGE, E. Redes sociales y asociaciones civiles taiwanesas en la Ciudad de Buenos Aires. 2010. Ponencia presentada. VI Jornadas de Investigación en Antropología Social, Universidad de Buenos Aires, Buenos Aires, 3 al 6 de agosto de 2010. 
BRETAL, E. ‘¡Ay, este chino habla castellano!’. El caso de los jóvenes inmigrantes de origen taiwanés en la ciudad de La Plata. 2005. Ponencia presentada. IV Jornadas de Sociología de la UNLP, Universidad Nacional de La Plata, La Plata, 23 al 25 de noviembre de 2005.

CASALS, P. Inserción de los supermercadistas chinos en la ciudad de Mar del Plata. Trabajo final para la matéria Sociología de las Migraciones. Buenos Aires: Universidad CAECE, 2009.

CHINA'S policy paper on Latin America and the Caribbean. 2008. Disponible en: <http://www.gov.cn/english/official/2008-11/05/content_1140347.htm>. Acceso el: 1 jul. 2014.

DENARDI, L. Categorías morales y trayectorias de inmigrantes taiwaneses en la Ciudad de Buenos Aires. Ser chino -en sentido amplio- y ser taiwanés. In: JORNADAS INTERDISCIPLINARIAS DE JÓVENES INVESTIGADORES EN CIENCIAS SOCIALES, 1., 2013, Buenos Aires. Mesa $n^{\circ} 10$ : perspectivas interdisciplinarias en el estudio de las categorías y los repertorios morales. Buenos Aires: Universidad Nacional de San Martín, 2013a. p. 16-27. Disponible en: <http://www.idaes.edu.ar/sitio/noticias/280/Mesa\%2010. pdf $>$. Acceso el: 1 jul. 2014.

DENARDI, L. Desconstruyendo prejuicios. Dang Dai, 1 jul. 2013b. Disponible en: <http://dangdai.com.ar/joomla/index.php?option=com_conte nt\&view=article\&id=3009:deconstruyendo-prejuicios\&catid=3:contribucion es\&Itemid=11>. Acceso el: 1 jul. 2013.

EDEL, M.; EDEL, A. Anthropology and ethics. Springfield: Charles C. Thomas, 1959.

FASSIN, D. Beyond good and evil?: Questioning the anthropological discomfort with morals. Anthropological Theory, London, v. 8, n. 4, p. 333344, Dec. 2008.

FLEISCHER, F. La diáspora china: un acercamiento a la migración china en Colombia. Revista de Estudios Sociales, Bogotá, n. 42, p. 71-79, 2012. 
FREIRE, J. Agir no regime de desumanização: esboço de um modelo para análise da sociabilidade urbana na cidade do Rio de Janeiro. Dilemas: Revista de Estudos de Conflito e Controle Social, Rio de Janeiro, v. 3, n. 10, p. 119142, 2010.

GELBER, H. El dragón y los demonios extranjeros: China y el mundo a lo largo de la historia. Barcelona: RBA Libros, 2008.

GIRALDO, G. ¿Qué explica ese cambio en el ranking? Tiempo, 11 mayo 2014. Disponible en: <http://tiempo.infonews.com/nota/6653/que-explicaese-cambio-en-el-ranking>. Acceso el: 12 mayo 2014.

GRIMSON, A.; NG, G.; DENARDI, L. Las organizaciones de inmigrantes chinos en Argentina. 2014. Mimeografiado.

GUERRAZAMPONI, C. La diáspora china. Buenos Aires: Consejo Argentino para las Relaciones Internacionales, 2010. Materiales de Conferencias, Seminarios y Reuniones de Trabajo, n. 6. Disponible en: <http://www.cari. org.ar/pdf/mcsrt6.pdf>. Acceso el: 1 jul. 2014.

HOWELL, S. The ethnography of moralities. London: Routledge, 1997.

LOUIE, A. Re-territorializing transnationalism: Chinese Americans and the Chinese motherland. American Ethnologist, Arlington, v. 27, n. 3, p. 645-669, 2000.

MONETA, C.; CESARÍN, S. Tejiendo redes: estrategias de las empresas transnacionales asiáticas en América Latina. Buenos Aires: Universidad Nacional Tres de Febrero, 2012.

NG, G.; DENARDI, L. El cultivo del entendimiento. Dang Dai, Buenos Aires, n. 9, p. 46-48, otoño 2014.

NOEL, G. De los códigos a los repertorios: algunos atavismos persistentes acerca de la cultura y una propuesta de reformulación. Revista Latinoamericana de Metodología de las Ciencias Sociales, Buenos Aires, v. 3, n. 2, 2013. 
ORDÓÑEZ DE PABLOS, P. La importancia del guanxi, renqing y xinyong en las relaciones empresariales en China: implicaciones para las empresas españolas. Revista de Economía, Madrid, n. 818, p. 221-234, 2004.

PAPPIER, A. Inmigración china en Argentina. El Barrio Chino de Buenos Aires como caso de estudio intercultural. In: CONGRESO DE LA ALADAA, 13., 2011, Bogotá. Memorias del... México: Asociación Latinoamericana de Estudios de Asia y África, 2011. Disponible en: <http://ceaa.colmex.mx/ aladaa/memoria_xiii_congreso_internacional/images/pappier.pdf $>$. Acceso el: 1 jul. 2014.

PINHEIRO MACHADO, R. Uma ou duas Chinas? A “questão Taiwan” sob o ponto de vista de uma comunidade chinesa ultramar (Ciudad del Este, Paraguai). Civitas, Porto Alegre, v. 10, n. 3, p. 468-489, 2010.

SASSONE, S.; MERA, C. Barrios de migrantes en Buenos Aires: identidad, cultura y cohesión socioterritorial. 2007. Ponencia presentada. V Congreso Europeo CEISAL de Latinoamericanistas, Bruselas, 11 al 14 de abril de 2007.

TORINO, M. La invasión silenciosa: los golden boys chinos desembarcan en la Argentina. Cronista.com, 25 jul. 2014. Disponible en: <http://www.cronista. com/3dias/La-invasion-silenciosa-los-golden-boys-chinos-desembarcan-enla-Argentina-20140725-0007.html>. Acceso el: 26 jul. 2014.

TREJOS, B.; CHIANG, N. Young taiwanese immigration to Argentina: the challenges of adaptatios, self identity and returning. International Journal of Asia-Pacific Studies, Pulau Pinang, v. 8, n. 2, p. 113-143, 2012.

WALDINGER, R.; TSENG, Y. Divergent diasporas: the Chinese communities of New York and Los Angeles compared. Revue Européenne des Migrations Internationales, Poitiers, v. 8, n. 3, p. 91-115, 1992.

WERNECK, A. Sociologia da moral como sociologia da agência. Revista Brasileira de Sociologia da Emoção, João Pessoa, v. 12, n. 36, p. 704-718, 2013. 
WU, D. Y. The construction of chinese and non chinese identities. Daedalus, Cambridge, v. 120, n. 2, p. 159-179, 1991.

YANG, M. M. Gifts, favors and banquets: the art of social relationship in China. Ithaca: Cornell University Press, 1994.

ZHANG, B. Of nonlimites locality/identity: Chinese diaspora poetry in America. Journal of American Studies, London, v. 40, n. 1, p. 133-153, 2006.

ZHANG, T. Inmigrantes chinos en Argentina, una comunidad joven, dinámica y amante de una convivencia armoniosa. Revista de la COPPPAL, Buenos Aires, p. 56-59, 2007.

ZUZEK, C. Identidad y aculturación: el caso de los inmigrantes taiwaneses jóvenes. Buenos Aires: Universidad del Salvador, 2004. Informe de proyecto. Instituto de Investigación en Ciencias Sociales, Facultad de Ciencias Sociales.

Recebido em: 31/08/2014 Aprovado em: 03/02/2015 